The Astrophysical Journal, 625:996-1003, 2005 June 1

(C) 2005. The American Astronomical Society. All rights reserved. Printed in U.S.A.

\title{
POLARIZATION OF L DWARFS BY DUST SCATTERING
}

\author{
Sujan Sengupta \\ Institute of Astronomy and Astrophysics, Academia Sinica, P.O. Box 23-141, Taipei 106, Taiwan; \\ and Indian Institute of Astrophysics, Koramangala, Bangalore 560 034, India; sujan@iiap.res.in \\ AND \\ Sun KwoK \\ Institute of Astronomy and Astrophysics, Academia Sinica, P.O. Box 23-141, Taipei 106, Taiwan; kwok@asiaa.sinica.edu.tw \\ Received 2004 November 3; accepted 2005 February 11
}

\begin{abstract}
The degree of polarization in L dwarfs of spectral types L0-L6 resulting from dust scattering in a rotationinduced oblate photosphere is calculated. Assuming that forsterite is the main condensate, we derive the atmospheric dust distribution for different spectral types based on a chemical equilibrium model. The degree of polarization at optical is then calculated using a single-scattering model. The expected linear polarization at optical is found to peak at around spectral type L1. For a fixed rotational velocity, the degree of polarization decreases from hotter to cooler objects. However, with the increase in mean grain size, the degree of linear polarization reduces significantly. We fit the recently observed linear polarimetric data of $L$ dwarfs and find that the single dust scattering model coupled with the chemical equilibrium models of condensates is consistent with the observational results.
\end{abstract}

Subject headings: dust, extinction — polarization — scattering — stars: atmospheres — stars: low-mass, brown dwarfs

\section{INTRODUCTION}

Observations of $\mathrm{L}$ dwarfs with effective temperatures of $1400-2200 \mathrm{~K}$ have led to the investigation of dust condensates in their atmospheres (Kirkpatrick et al. 1999). Because of complete gravitational settling, grains are expected to condense beyond the visible atmosphere for objects with effective temperatures below $1400 \mathrm{~K}$. At higher effective temperatures, grains can be present in the visible atmosphere because of incomplete gravitational settling (Burrows \& Sharp 1999; Burrows et al. 2001; Ackerman \& Marley 2001; Allard et al. 2001; Tsuji et al. 2004, 1996).

Using a thermodynamical model based on homogeneous and heterogeneous condensation assumptions, Cooper et al. (2003, hereafter $\mathrm{C} 03$ ) were able to determine the distribution of different species of dust particles. Using solutions of dust moment equations in a static atmosphere, Woitke \& Helling (2003, 2004) studied the continuous nucleation of solid particles from the gas phase. By imposing the requirement of minimum mixing activities and thermodynamical stability, they were able to determine the upper boundary (called "cloud deck") and the lower boundary (called "cloud base").

The possibility of detecting polarization at optical from grains in the atmospheres of L dwarfs was first raised by Sengupta \& Krishan (2001). Fast rotation of L dwarfs (Basri et al. 2000) will induce the shape of their photosphere into the form of an oblate ellipsoid, and this nonsphericity will lead to incomplete cancellation of the polarization from different areas of the stellar surface. This prediction was first confirmed by the detection of linear polarization at $768 \mathrm{~nm}$ from a few $\mathrm{L}$ dwarfs by Menard et al. (2002). Recently Zapatero Osorio et al. (2005) have reported $R$ - and $I$-band detection of linear polarization from several $\mathrm{L}$ dwarfs. Since polarization in the optical is unlikely to be due to Zeeman splitting of atomic or molecular lines or by synchrotron radiation, the observed polarization can best be explained by single dust scattering in a rotationally induced oblate atmosphere (Sengupta 2003).
The observed polarization of DENIS-P J0255-4700 and 2MASS J0036+1821 was modeled by Sengupta (2003) by assuming minimum oblateness of these objects based on a model of complete degeneracy of a nonrelativistic polytropic gas under hydrostatic equilibrium. The observed degree of polarization was obtained by considering different parameters for the scale height, number density, and mean radius of the grains. By minimizing the oblateness and the number density of grains, an upper limit on the mean grain radius that is consistent with the polarization observed by Menard et al. (2002) was obtained. However, the process of dust condensation was not considered.

In the present paper, we present detailed theoretical models of optical linear polarization from L dwarfs of spectral types ranging from $\mathrm{L} 0$ to $\mathrm{L} 6$ based on the dust condensation model of C03. With this model, we fit the observed polarization from several L dwarfs and predict the amount of polarization expected from any $\mathrm{L}$ dwarfs with given rotational velocity.

In $\S 2$, we present the formalism for the calculation of polarization from single dust scattering in an oblate medium. In $\S 3$, the adopted atmospheric model is described. The estimation of rotationally induced oblateness is presented in $\S 4$, and the dust model parameters are described in $\S 5$. The results and discussions are presented in $\S 6$, followed by conclusions in $\S 7$.

\section{POLARIZATION BY SINGLE DUST SCATTERING}

If a stellar object is perfectly spherical then the net polarization would be zero because of the cancellation of the contribution of each point on the photosphere. The observation of nonzero polarization from a few $\mathrm{L}$ dwarfs therefore suggests that the scattering geometry is asymmetric, which could be the result of fast rotation of the objects. Since the dust density is low and scattering by atoms and molecules does not contribute to polarization significantly, the single-scattering approximation is reasonable for the region where the optical depth $\tau<1$. If present, multiple scattering can reduce the degree of polarization by a few orders of magnitude (Sengupta \& Krishan 2001) 
because the planes of the scattering events are randomly oriented and average each other's contribution out from the final polarization. The effects of oblateness of an object on linear polarization due to single scattering by spherical grains have been discussed by Dolginov et al. (1995) and by Simmons (1982). In this paper, we adopt the formalism given by Simmons (1982).

For an optically thin atmosphere, the total linear polarization $p(k)$ can be written as

$$
p(k)=|Z(k)|=\left|Z^{*}(k)\right|
$$

where $k=2 \pi / \lambda, \lambda$ being the wavelength, the asterisk denotes the complex conjugate, and

$Z(k)=\frac{1}{k^{2}} \iiint \frac{i_{1}(\theta, k)-i_{2}(\theta, k)}{2} n(r, \theta, \phi) e^{2 i \phi} d \omega d r$,

where $d w$ is the element of solid angle. In the above equation, $\theta$ is the scattering angle, $n$ is the number density of scattering particles, and $i_{1}$ and $i_{2}$ are the scattering functions given by (van de Hulst 1957)

$$
i_{1}(\theta)=\left|\sum_{n=1}^{\infty} \frac{2 n+1}{n(n+1)}\left[a_{n} \pi_{n}(\theta)+b_{n} \tau_{n}(\theta)\right]\right|^{2}
$$

and

$$
i_{2}(\theta)=\left|\sum_{n=1}^{\infty} \frac{2 n+1}{n(n+1)}\left[b_{n} \pi_{n}(\theta)+a_{n} \tau_{n}(\theta)\right]\right|^{2}
$$

where

$$
\pi_{n}(\theta)=-\frac{1}{\sin \theta} P_{n}^{1}(\cos \theta)
$$

and

$$
\tau_{n}(\theta)=-\frac{d}{d \theta} P_{n}^{1}(\cos \theta)
$$

The coefficients $a_{n}$ and $b_{n}$ are in general complex functions of the refractive index $m$ and the particle radius to wavelength ratio.

For a smooth density distribution, one can write

$$
n(r, \theta, \phi)=\sum_{l=0}^{\infty} \sum_{m=-l}^{m=l} n_{l m}(r) Y_{l m}(\theta, \phi),
$$

where

$$
\begin{gathered}
Y_{l m}(\theta, \phi)=\alpha(l, m) P_{l}^{m}(\cos \theta) \exp (\operatorname{im} \phi), \\
\alpha(l, m)=\left[\frac{(2 l+1)(l-m)}{4 \pi(l+m)}\right]^{1 / 2},
\end{gathered}
$$

and $P_{l}^{m}$ is the associated Legendre function of the first kind.

Substituting equation (7) into equation (2) and integrating over $\phi$, we get

$$
p(k)=\frac{2 \pi}{k^{2}} \sum_{l=2}^{\infty} N_{l 2} F_{l 2}
$$

where

$$
F_{l m}=\alpha(l, m) \int_{-1}^{1} \frac{i_{1}-i_{2}}{2} P_{l}^{m}(\cos \theta) d(\cos \theta) .
$$

Considering an axisymmetry density distribution with a rotational invariance around some axis and using the addition theorem of spherical harmonic, $N_{l m}$ can be written as

$$
\begin{aligned}
N_{l m}= & 2 \pi \alpha(l, m) P_{l}^{m}\left(\cos \theta_{i}\right) e^{-2 i \phi} \\
& \times \int_{R_{2}}^{R_{1}} n(r) d r \int_{-1}^{1} \frac{P_{l}(\mu) d \mu}{\left[1+\left(A^{2}-1\right) \mu^{2}\right]^{1 / 2}},
\end{aligned}
$$

where $R_{1}$ and $R_{2}$ are the outer and the inner equatorial axis lengths, respectively, $A$ is the ratio of the length of the equatorial axis to the polar axis, $\theta_{i}$ is the viewing angle of the observer, and $\mu=\cos \theta$.

At an edge-on view, $\theta_{i}=\pi / 2$ and $\phi=0$, and hence $N_{l m}$ is real. From the equation of hydrostatic equilibrium, we have an expression of the particle density as a function of gas pressure:

$$
n(r) d r=\frac{n(P) d P}{g \rho(P)}
$$

where $P$ is the pressure at different geometrical height, $\rho$ is the mass density at different pressure scale, and $g$ is the surface gravity (which can be assumed to be constant for a geometrically thin atmosphere). Substituting equations (11) and (12) into equation (10), we have

$$
\begin{aligned}
p(\lambda)= & \frac{\lambda^{2}}{g} \int_{P_{1}}^{P_{2}} \frac{n(P) d P}{\rho(P)} \\
& \times \sum_{l=2}^{\infty}\left\{\alpha^{2}(l, m) P_{l}^{m}(0) F_{l 2} \int_{-1}^{1} \frac{P_{l}(\mu)}{\left[1+\left(A^{2}-1\right) \mu^{2}\right]^{1 / 2}} d \mu\right\}
\end{aligned}
$$

for the degree of polarization for a hydrostatic atmosphere viewed edge-on.

\section{THE ATMOSPHERIC MODELS}

The effective temperature of the L dwarfs of different spectral type can be approximated by the linear empirical relationship of Stephens et al. (2001):

$$
T_{\text {eff }}=2220-100 L,
$$

where $L$ is the spectral type between L0 and L8. Recently Vrba et al. (2004) and Golimowski et al. (2004) have presented $T_{\text {eff }}$ measurements for $\mathrm{L}$ and $\mathrm{T}$ dwarfs based on bolometric luminosities. In the present work we adopt the sixth-order polynomial fit given by Golimowski et al. (2004) for translating the $T_{\text {eff }}$ into spectral type. Figure 1 shows the $T_{\text {eff }}$ for different spectral types calculated by using the linear relationship given by Stephens et al. (2001) and by the polynomial formula provided by Golimowski et al. (2004). The $T_{\text {eff }}$ calibration of Vrba et al. (2004) and Golimowski et al. (2004) agree well in the interval L3-L8, but there are significant differences in earlier types. In our calculations for the degree of polarization, the effective temperature $T_{\text {eff }}$ is used, and hence the degree of polarization should be considered strictly as a function of $T_{\text {eff }}$ rather than the spectral type. 


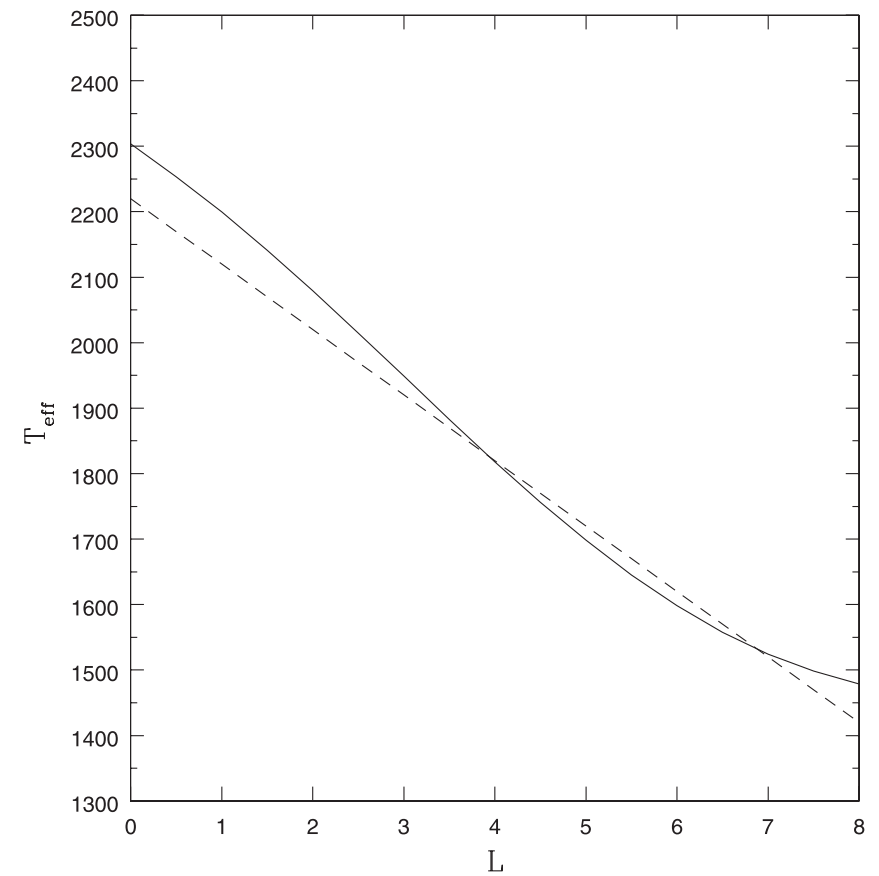

FIG. 1.-Effective temperature vs. spectral type: the solid line represents the calibration of $T_{\text {eff }}$ using the sixth-order polynomial fit as given in Golimowski et al. (2004), while the dashed line represents that using the linear relationship given in Stephens et al. (2001).

The surface gravity of L dwarfs older than about a few hundred million years varies from $g=10^{5}$ to $3 \times 10^{5} \mathrm{~cm} \mathrm{~s}^{-2}$. Evolutionary models by Chabrier et al. (2000) show younger L dwarfs to have surface gravity smaller than $10^{5} \mathrm{~cm} \mathrm{~s}^{-2}$. In this paper, we assume $g=10^{5} \mathrm{~cm} \mathrm{~s}^{-2}$.

Formation of dust makes it a prohibitive task to develop a fully consistent atmospheric model for ultra-cool dwarfs. This is mainly because the presence of a dust cloud affects the radiative equilibrium of the upper atmosphere and hence alters the $T-P$ profile from that of a cloud-free atmosphere. On the other hand, the $T$ - $P$ profile dictates the position and the chemical equilibrium of condensates. Allard et al. (2001) presented atmospheric models for two limiting cases, e.g., one with inefficient gravitational settling wherein the dust is distributed according to chemical equilibrium predictions (AMES-dusty) and another with efficient gravitational settling in which situation dust has no effect on the thermal structure (AMES-cond). Tsuji et al. (2004) have proposed a unified cloudy model (UCM) in which the segregation of dust from the gaseous mixture takes place in all the ultracool dwarfs and at about the same critical temperature.

In the present work, the temperature-pressure profiles for the L dwarfs with different spectral type are calculated by solving the non-LTE radiative transfer equations coupled with the hydrostatic equilibrium equations. We have employed the full Mie theory, incorporating the dust opacity as well as the Mie phase function. The calculations are first performed by taking only gas opacities. We have adopted the atmospheric opacity sources discussed in Saumon et al. (2000) and assumed solar metallicity. The $T-P$ profile thus obtained determined the position of the cloud. We then incorporated dust opacities to calculate the final set of $T-P$ profile. This $T-P$ profile is then used again to determine the base and the deck of the cloud. Our pressuretemperature profiles are checked against those presented in Burrows et al. (2001) and in C03, as well as models kindly provided by M. Marley (1999, private communication). Nevertheless, the polarization profile would change depending on the chemical equilibrium procedure adopted in different atmospheric models.

\section{ROTATION-INDUCED OBLATENESS}

The observation of nonzero polarization from unresolved objects indicates the nonsphericity of the photosphere. The nonsphericity may result from several causes. For example, rotation of a stellar object will result in a shape of an oblate ellipsoid, as is evident in the outer solar planets. At a 1 bar pressure level, the eccentricity of Jupiter, Saturn, and Uranus are $0.35,0.43$, and 0.21 , respectively. Apart from rotational effects, tidal interaction with the companion in a binary system also imposes an ellipsoidal shape extending toward the companion.

Spectroscopic studies by Basri (1999) and Basri et al. (2000) indicate rapid rotation of ultracool dwarfs along their axis. The brown dwarf Kelu 1 is found to be the fastest rotator with a projected angular velocity $(v \sin i)$ as high as $60 \mathrm{~km} \mathrm{~s}^{-1}$. The observation of optical polarization from L dwarfs with known projected angular velocity implies nonsphericity of the photosphere due to rotation.

The oblateness of a rotating object has been discussed by Chandrasekhar (1933) in the context of polytropic gas configuration under hydrostatic equilibrium. For a slow rotator, the relationship for the oblateness $f$ of a stable polytropic gas configuration under hydrostatic equilibrium is given by

$$
f=\frac{2}{3} C \frac{\Omega^{2} R_{e}^{3}}{G M}
$$

where $M$ is the total mass, $R_{e}$ is the equatorial radius, and $\Omega$ is the angular velocity of the object. $C$ is a constant whose value depends on the polytropic index. For the polytropic index $n=0$, the density is uniform and $C=1.875$. This configuration is known as the Maclaurin spheroid. For a polytropic index of $n=1.0, C=1.1399$, which is appropriate for Jupiter (Hubbard 1984). For nonrelativistic, completely degenerate gas, $n=1.5$ and $C=0.9669$. The rotationally induced oblateness of solar planets has been discussed in detail by Hubbard (1984) and Murray \& Dermott (2000). Recently, the formalism for oblateness was extended to extrasolar planets by Barnes \& Fortney (2003), who used the Darwin-Radau relationship

$$
f=\frac{\Omega^{2} R_{e}^{3}}{G M}\left[\frac{5}{2}\left(1-\frac{3}{2} K\right)^{2}+\frac{2}{5}\right]^{-1}
$$

(Murray \& Dermott 2000; Barnes \& Fortney 2003) to relate rotation to oblateness. In equation (17), $K=I / M R_{e}^{2} \leq 2 / 3$ is the moment of inertia parameter of an object with moment of inertia I. The Darwin-Radau relationship is exact for uniform density objects $(K=0.4)$ and provides a reasonable (within errors of a few percent) estimation of the oblateness of the solar planets.

Since L dwarfs have an extended convective region and their moment of inertia is an undetermined parameter, we adopt the relationship given by Chandrasekhar with the polytropic index $n=1.0$ and 1.5. A value of $n<1.0$ would yield too low a density for brown dwarfs, whereas $n=1.5$ would provide minimum possible oblateness due to rotation. We have calculated the mass and radius of $\mathrm{L}$ dwarfs of different spectral types by adopting the empirical relationship given in Marley et al. (1996).

\section{THE DUST PARAMETERS}

The dust distribution in the atmosphere is calculated based on the one-dimensional cloud model of $\mathrm{C} 03$. This model assumes 


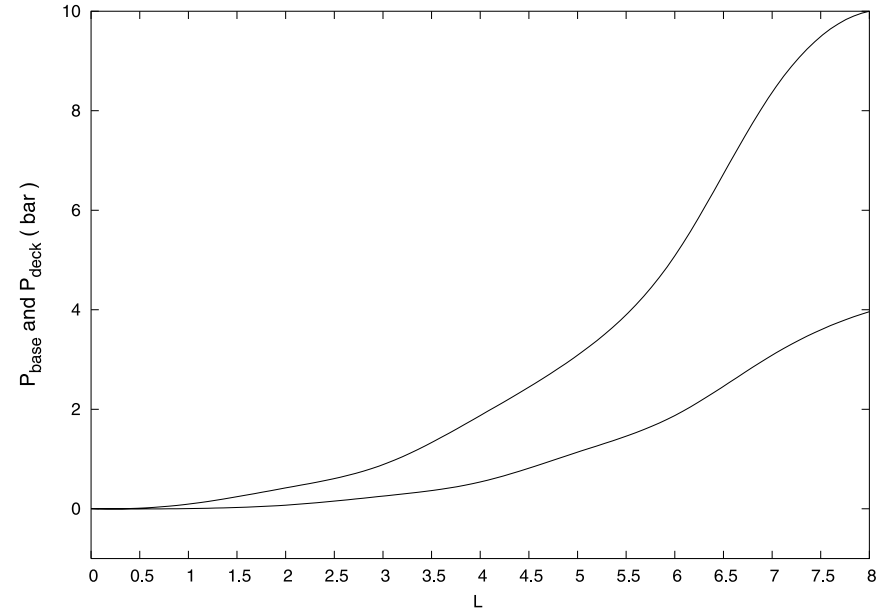

FIG. 2.-Locations of forsterite cloud base (top curve) and cloud deck (bottom curve) for different spectral type L0-L8.

chemical equilibrium throughout the atmosphere and uniform density distribution across the surface of an object at each given pressure and temperature. Under these assumptions, the number density of cloud particles is given by

$$
n(P)=q_{c}\left(\frac{\rho}{\rho_{d}}\right)\left(\frac{\mu_{d}}{\mu}\right)\left(\frac{3}{4 \pi a^{3}}\right),
$$

where $\rho$ is the mass density of the surrounding gas, $a$ is the cloud particle radius, $\rho_{d}$ is the mass density of the dust condensates, and $\mu$ and $\mu_{d}$ are the mean molecular weight of atmospheric gas and condensates, respectively. The condensate mixing number ratio $\left(q_{c}\right)$ is given as

$$
q_{c}=q_{\text {below }} \frac{P_{c, l}}{P}
$$

for heterogeneously condensing clouds. In the above equation, $q_{\text {below }}$ is the fraction of condensible vapor just below the cloud base, $P_{c, l}$ is the pressure at the condensation point (presented graphically in Fig. 1 of $\mathrm{C} 03$ ), and $P$ is the gas pressure in the atmosphere. As in $\mathrm{C} 03$, we employ the ideal gas equation of state $\rho=\mu P / R T$ to relate the gas mass density $\rho$ to the atmospheric temperature $T$ and pressure $P . R$ is the universal gas constant and $\mu=2.36$ for solar composition gas in which hydrogen is present in the molecular state.

The kinds of solid condensates possible in the atmospheres of $\mathrm{L}$ dwarfs include forsterite $\left(\mathrm{Mg}_{2} \mathrm{SiO}_{4}\right)$, gehlenite $\left(\mathrm{Ca}_{2} \mathrm{Al}_{2} \mathrm{SiO}_{7}\right)$, and iron (C03), as well as $\mathrm{TiO}_{2}$ (Woitke \& Helling 2004). However, forsterite forms in abundance in the atmosphere and plays the most crucial role in governing the continuum spectrum. The other species such as gehlenite are much less abundant than forsterite (about a factor of 10). In the present work, we consider only forsterite. The effect of other species on the degree of polarization is discussed later. We have used the data from C03 for forsterite: $\mu_{d}=140.7 \mathrm{~g} \mathrm{~mol}^{-1}, \rho_{d}=3.2 \mathrm{~g} \mathrm{~cm}^{-3}$, and $q_{\text {below }}=3.2 \times 10^{-5}$ (assuming a solar abundance distribution of the elements as discussed in C03). Throughout our investigation, we have considered the wavelength $\lambda=0.850 \mu \mathrm{m}$, as the observed polarimetric data presented by Zapatero Osorio et al. (2005) is obtained at $I$ band centered at this wavelength. The object 2MASS J0036+1820 is observed by Zapatero Osorio et al. (2005) at $R$ band (centered at $0.641 \mu \mathrm{m}$ ) as well as at $I$ band (centered at $0.85 \mu \mathrm{m}$ ). The same object is observed by Menard et al. (2002) at $I$ band (centered at $0.768 \mu \mathrm{m}$ ). Therefore, for this

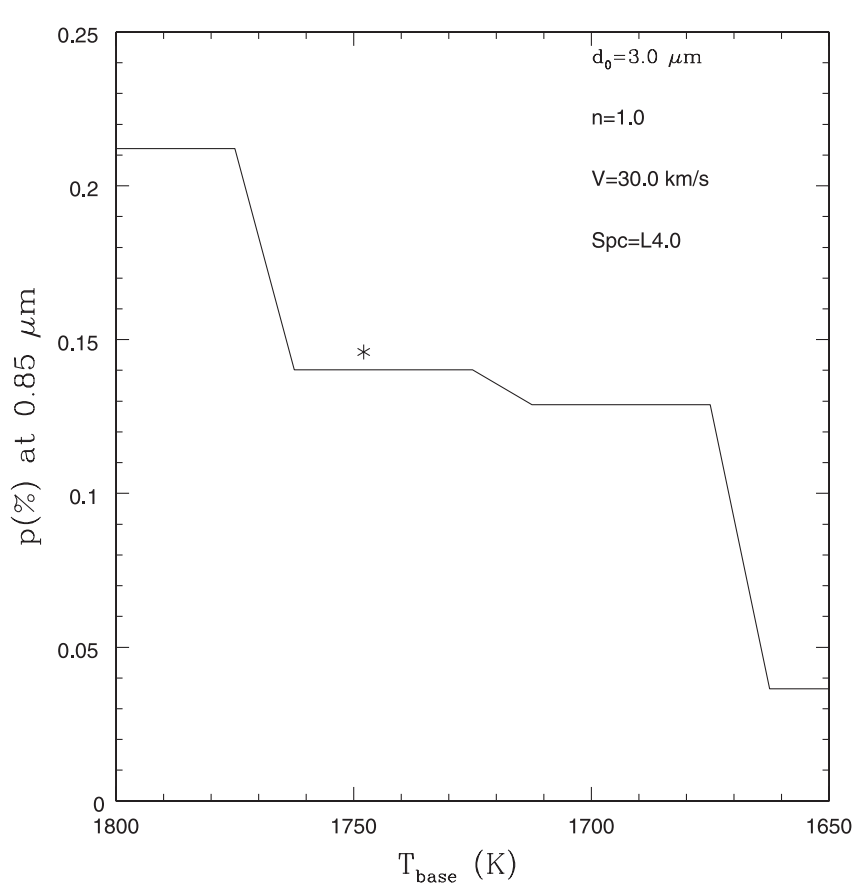

FIG. 3.-Degree of polarization for an L4.0 object with the cloud base situated at different temperature in the atmosphere but with a fixed particle number density. The asterisk indicates the degree of polarization when the cloud base is situated at $1750 \mathrm{~K}$ as determined from the intersection of the condensation curve and the $T-P$ curve for L4.0 with $g=10^{5} \mathrm{~cm} \mathrm{~s}^{-2}$.

object we model the degree of polarization at different wavelengths. The real part of the refractive index is fixed at 1.65 , and the imaginary part is taken by interpolating the data given in Scott \& Duley (1996). It should be mentioned that the refractive index of amorphous condensates might differ under different physical conditions.

Apart from the calculation of the grain number density, the location of the cloud in the atmosphere plays an important role in determining the amount of polarization. The location of the cloud base for different atmospheric models and different chemical species is determined by the intersection of the $T-P$ profile of the atmosphere model and the condensation curve $P_{c, l}$ as prescribed in $\mathrm{C} 03$. Taking the condensation curve for forsterite, we determine the base of the cloud for each spectral type, from L0 to L8. Figure 2 presents the atmospheric pressure height at which the cloud base is situated for models with different spectral types, and Figure 3 shows how the degree of polarization alters with the change in the location of the cloud base. As the effective temperature decreases from $\mathrm{L} 0$ to $\mathrm{L} 8$, the cloud base is pushed deeper into the atmosphere. According to the condensation curve given in $\mathrm{C} 03$, the base of forsterite cloud for an L8 object $\left(T_{\text {eff }} \simeq 1480 \mathrm{~K}\right.$ ) is situated at about a 10.0 bar pressure height when the surface gravity of the object is assumed to be $10^{5} \mathrm{~cm} \mathrm{~s}^{-2}$. For a similar model, Woitke \& Helling (2004) found the base of $\mathrm{TiO}_{2}$ at 9.4 bar pressure height. Similarly, for an L4 ( $\left.T_{\text {eff }} \simeq 1820 \mathrm{~K}\right)$ object, the forsterite cloud base is found to be situated at 2.2 bar, while Woitke \& Helling (2004) found it to be at 2.7 bar for $\mathrm{TiO}_{2}$ cloud.

Theoretical investigation (Tsuji 2005) claims that the thickness of the dust clouds and hence the location of the cloud deck influences the spectral energy distribution of $\mathrm{L}$ and $\mathrm{T}$ dwarfs. Also, it is suggested that the vertical height of dust cloud may vary for a given $T_{\text {eff }}$ and surface gravity (Knapp et al. 2004; Tsuji 2005). The degree of polarization too is strongly dependent on the vertical height of the dust cloud and hence on the 
location of the cloud deck. In C03, the cloud deck is considered to be at one scale height above the base. The condensation curve $P_{c, l}$ decreases exponentially with the decrease in the atmospheric temperature and the value of $P_{c, l}$ becomes negligibly small at $T=1600 \mathrm{~K}$. For different atmospheric models, this temperature is attained at different atmospheric pressure height. The position of the cloud deck for different spectral types is also presented in Figure 2. From Figure 2 we note that the thickness of the cloud decreases as one goes from L8 to L0. In other words, the thickness of the dust cloud decreases with the increase in effective temperature for a fixed value of surface gravity. For L dwarfs hotter than L2, the cloud is very thin, much less than one scale height. For an L8 object, the forsterite cloud deck is calculated to be situated at about 4 bar pressure height. For a similar atmospheric model, Woitke \& Helling (2004) calculated the $\mathrm{TiO}_{2}$ cloud deck at 0.24 bar. For an L4 object the forsterite cloud deck in our model is at 0.5 bar pressure height, while the $\mathrm{TiO}_{2}$ cloud deck calculated by Woitke \& Helling (2004) for a similar object is at 0.1 bar. Therefore, the cloud thickness in our model is smaller than that of Woitke \& Helling (2004). The number density of $\mathrm{TiO}_{2}$ grains for L4 and L8 objects is presented graphically in Woitke \& Helling (2004).

At present, there is no convincing justification in favor of any specific form of the particle size distribution function. C03 considered a particle size distribution function that is consistent with the measurements of grain distribution attained in Earth's water clouds, while Ackerman \& Marley (2001) and Saumon et al. (2000) considered a broad lognormal size distribution. In the present work, we adopt the size distribution function given by the latter authors, which is expressed as

$$
f(d)=\frac{d}{d_{0}} \exp \left[-\frac{\ln ^{2}\left(d / d_{0}\right)}{\ln ^{2} \sigma}\right],
$$

where $d$ is the diameter of the particles, $d_{0}$ is the mean diameter, and $\sigma$ is fixed at 1.3, which provides a good fit to the red spectrum of L dwarfs.

\section{RESULTS AND DISCUSSION}

In a heterogeneously condensing cloud model with forsterite as the dominant constituent, the number density of grains, the cloud base, and its vertical scale height are fixed. The remaining free parameters are the mean particle diameter $d_{0}$ and the oblateness induced by rotation. As discussed by $\mathrm{C} 03$, the particle sizes significantly vary with the effective temperature, surface gravity, and vertical height of the dust cloud. In the present work we have calculated the degree of polarization for a fixed surface gravity $g=10^{5} \mathrm{~cm} \mathrm{~s}^{-1}$. Figure 4 shows how the degree of polarization is altered with different mean particle diameter for objects with different spectral type and hence for different effective temperature. In a multiple-scattering scenario, it is important to consider different mean particle size at different pressure scale. However, in a single scattering, the photon scatters only with one particle, and therefore, for a fixed effective temperature, a fixed mean particle size is sufficient. This means that at every atmospheric pressure level, we use the lognormal distribution with particle mean diameter $d_{0}$.

We have included contributions to polarization by multipoles $l=2,4$, and 6 in equation (14). However, it is found that at optical wavelengths and for the particle size needed to account the observed polarization, contribution from $l=2$ is dominant over that of higher values for $l$.

The projected rotational velocity for several $\mathrm{L}$ dwarfs has been determined from observational data. In the absence of any

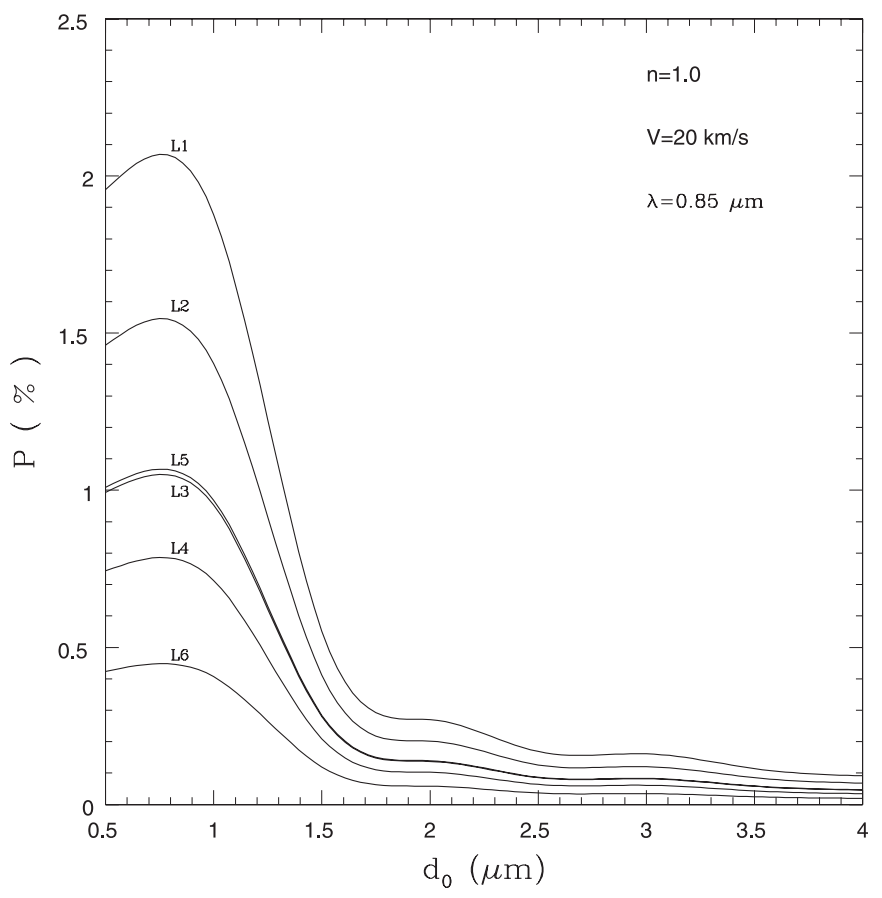

FIG. 4.-Degree of polarization as a function of mean grain diameter $d_{0}$ for L dwarfs with different spectral type (L1-L6).

knowledge on the inclination angle, the actual rotational velocity cannot be determined, yielding uncertainties in the oblateness. In the present work we have considered rotational velocity of $15,25,30,40$, and $50 \mathrm{~km} \mathrm{~s}^{-1}$. Figures 5 and 6 show how the degree of polarization significantly increases with the increase in rotational velocity.

A comparison of the degree of polarization presented in Figures 5 and 6 shows that if the mean particle diameter is increased, the degree of polarization decreases significantly because with the increase in grain size, the grain number density reduces according to equation (18).

As mentioned in $\S 5$, the location of the cloud base plays an important role in determining the degree of polarization. As one moves from L0 to L8, the cloud base goes deeper in the atmosphere owing to the decrease in effective temperature. This makes the vertical size of the cloud larger (Fig. 2) and hence substantial increase in the total number of dust grains. In Figure 3, we show how the degree of polarization is altered when the cloud base is changed but the particle number density remains unaltered. For an object of spectral type L4.0 ( $\left.T_{\text {eff }} \simeq 1820 \mathrm{~K}\right)$ with surface gravity $g=10^{5} \mathrm{~cm} \mathrm{~s}^{-2}$, the cloud base should be situated at an atmospheric temperature about $1750 \mathrm{~K}$. Keeping the particle number density the same as that calculated for the L 4 object, we calculated the degree of polarization by varying the location of the cloud base. The degree of polarization is found to increase as the location of the cloud base is pushed deeper into the atmosphere, where the temperature increases from 1600 to $1800 \mathrm{~K}$. This implies that as one moves from L8 to L0, that is, from cooler to hotter dwarfs, the degree of polarization decreases owing to the decrease in the vertical height of the dust cloud.

As mentioned in $\S 1$, Menard et al. (2002) for the first time detected linear polarization from three L dwarfs. They use the $I$-band Bessel filter having the central wavelength at $0.768 \mu \mathrm{m}$. Out of the three objects that show confirmed polarization, one object (DENIS-P J0255-4700) belongs to the spectral type L8 and has high rotational velocity $\left(40 \mathrm{~km} \mathrm{~s}^{-1}\right)$. This object shows a linear polarization of $0.167 \%$. On the other hand, Zapatero 


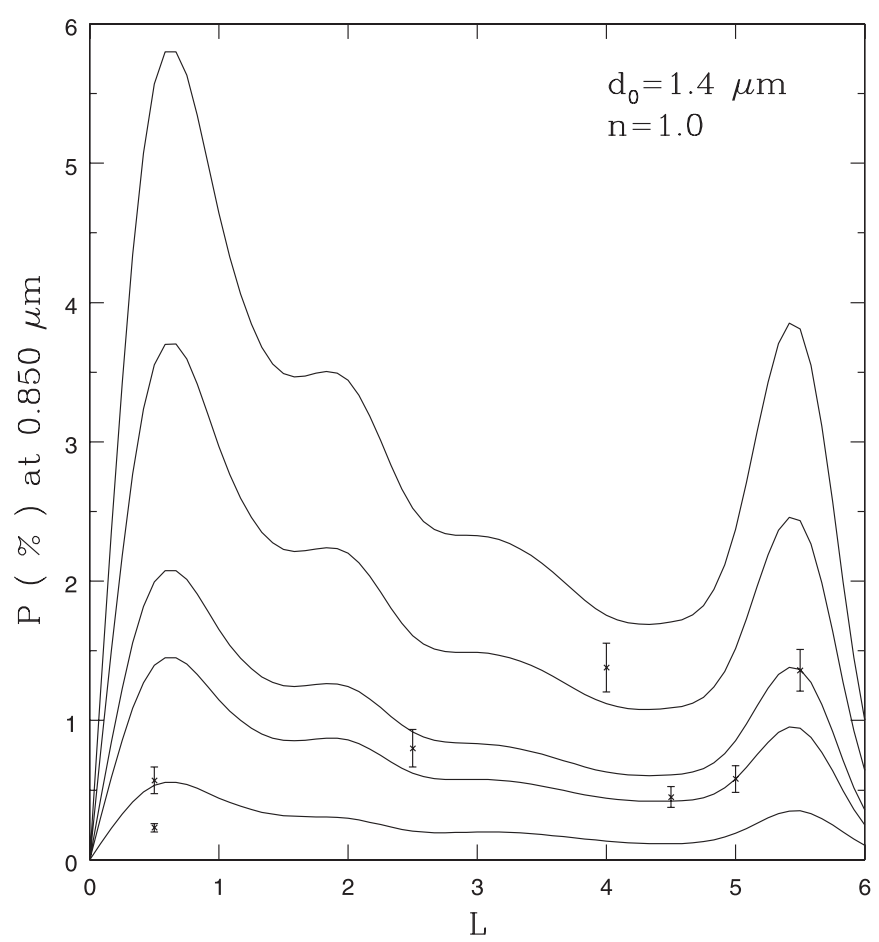

FIG. 5.-Degree of polarization for objects with different spectral type with the mean grain diameter $d_{0}=1.4 \mu \mathrm{m}$ and the polytropic index $n=1.0$. The five curves are for rotational velocities of $V=15,25,30,40$, and $50 \mathrm{~km} \mathrm{~s}^{-1}$, from bottom to top. The observed polarization of seven $\mathrm{L}$ dwarfs are plotted with their respective error bars.

Osorio et al. (2005) have reported confirmed polarization from nine L dwarfs in $I$ band (centered at $0.85 \mu \mathrm{m}$ ) and one L dwarf (2MASS $\mathrm{J} 0036+1821$ ) in $I$ as well as in $R$ band (centered at $0.641 \mu \mathrm{m})$. The object 2MASS J0036 +1821 is also observed by Menard et al. (2002) in $I$ band but at a different central wavelength. Hence, the degree of polarization at three different wavelength regions is available for this object.

Among the $10 \mathrm{~L}$ dwarfs that are found to show confirmed polarization by Zapatero Osorio et al. (2005), one (2MASS $\mathrm{J} 2244+20$ ) is reported to be significantly redder than those of other mid-L to late-L dwarfs in near-infrared and infrared. Out of the remaining nine L dwarfs, the projected rotational velocity of only four objects is known. The object 2MASS J2252-17 is of spectral type L7.5. Although its rotational velocity is not known, it shows a degree of polarization as high as $0.62 \%$. Unless the rotational velocity of this object is very high (even higher than the fastest L dwarf Kelu 1), the large difference in the observed degree of polarization from this object and DENIS-P J0255-4700 cannot be explained, as the mean particle size in the atmosphere of L dwarfs of same spectral type should not differ much. Further theoretical investigation on the distribution and location of condensates in the coolest L dwarfs are needed before modeling their degree of polarization. Hence, in the present work we discuss linear polarization of $\mathrm{L}$ dwarfs of spectral type ranging from L0 to L6.

Figure 5 shows the degree of linear polarization at $\lambda=$ $0.850 \mu \mathrm{m}$ for objects with different spectral types but fixed surface gravity $10^{5} \mathrm{~cm} \mathrm{~s}^{-2}$. It is found that the observed degree of polarization of several L dwarfs can be fitted if the mean diameter of grain is taken as $1.4 \mu \mathrm{m}$ and with the polytropic index $n=1.0$. The figure shows that the change in polarization with the spectral type is not linear, but overall the degree of polarization decreases as one moves from L1 to L6. The degree of

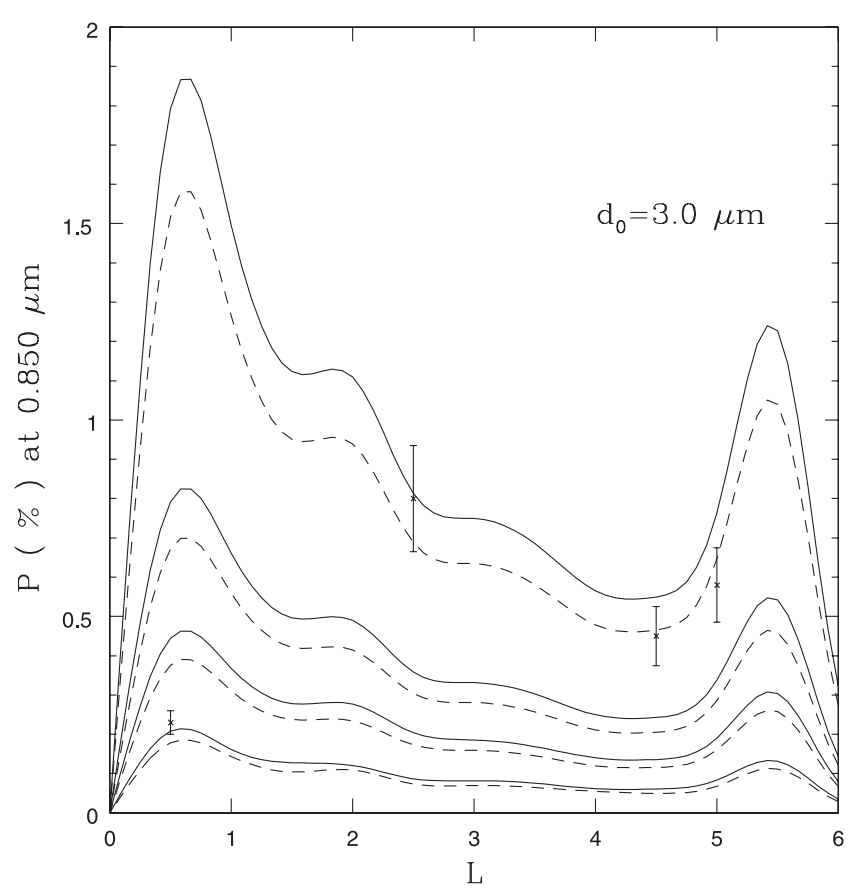

FIG. 6.-Same as Fig. 4 but with the mean grain diameter $d_{0}=3.0 \mu \mathrm{m}$. The solid curves represent the degree of polarization with the polytropic index $n=1.0$, while the dashed curves represent that with $n=1.5$. The four pairs of curves are for rotational velocities of $V=20,30,40$, and $60 \mathrm{~km} \mathrm{~s}^{-1}$, from bottom to top. The observed polarization of four $\mathrm{L}$ dwarfs is also presented.

polarization decreases substantially for objects hotter than L1 because condensation is not favored at such high temperature. The location of the cloud base shifts to a deeper region of the atmosphere as the effective temperature decreases and hence should cause an increase in the polarization (Fig. 3). However, the total amount of condensing material is conserved. Therefore, as the scale height of the cloud layer becomes smaller, the particle number density becomes higher, yielding higher polarization. Beyond the L1 object, the temperature becomes too high to favor condensation and the polarization falls rapidly to zero.

Zapatero Osorio et al. (2005) detected the polarization in the Johnson $R$ - and $I$-band filters centered on 0.641 and $0.850 \mu \mathrm{m}$, respectively, with the passband of these filters as 0.158 and $0.15 \mu \mathrm{m}$. Menard et al. (2002) detected the polarization in the Bessel $I$-band filter on $0.768 \mu \mathrm{m}$ with the passband as $0.138 \mu \mathrm{m}$. We have calculated the degree of polarization at the central wavelengths. The change in degree of polarization over the spread of wavelength should by and large be absorbed in the error bars.

Both 2MASS J1707+43 and 2MASS J1412+16 are of spectral type L0.5. 2MASS J1412+16, having projected rotational velocity $16.4 \mathrm{~km} \mathrm{~s}^{-1}$, shows a degree of polarization of $0.57 \pm$ 0.19 , while 2 MASS J1707+43 shows a degree of polarization of $0.23 \pm 0.06$, but its rotational velocity is not known. Figure 5 shows that the observed data of these two objects can be explained well if the rotational velocity of 2 MASS J1707+43 is much less than $15 \mathrm{~km} \mathrm{~s}^{-1}$. The model assumes that the mean diameter of grain is $1.4 \mu \mathrm{m}$ and the polytropic index $n=1.0$. The same model can explain the observed polarization of 2MASS J1507-16 (L5 with projected rotational velocity $27.2 \mathrm{~km} \mathrm{~s}^{-1}$ ) if its effective temperature is slightly higher than that given by the spectral type$T_{\text {eff }}$ polynomial formula. We put it as L5.5 to show that if the effective temperature of this object corresponds to spectral type L5.5 in the polynomial formula then the observed polarization can be well fitted. The same model can explain the observed 
polarization from 2MASS J2158-15 (L4), 2MASS J0141+18 (L4.5), and 2MASS J0144-07 (L5) if their rotational velocities are about 45,25 , and $25 \mathrm{~km} \mathrm{~s}^{-1}$, respectively. However, this model fails to fit the data from Kelu 1 (L2.5) unless its actual rotational velocity is between 25 and $30 \mathrm{~km} \mathrm{~s}^{-1}$.

Figure 6 presents the degree of polarization with the same model but with larger mean particle diameter. It is found that the observed degree of polarization from 2MASS 1707+43 (L0.5) can also be explained if its rotational velocity is $20 \mathrm{~km} \mathrm{~s}^{-1}$ but the atmosphere contains grains with mean diameter $3.0 \mu \mathrm{m}$. However, the mean grain size of objects with the same spectral type should not differ much, and therefore we predict that the rotational velocity of this object is about $5-10 \mathrm{~km} \mathrm{~s}^{-1}$ with mean particle diameter $1.4 \mu \mathrm{m}$ as presented in Figure 5. Figure 6 shows that the observed polarization of Kelu 1 (L2.5) can be explained if the mean particle diameter is $3.0 \mu \mathrm{m}$. However, it is worth mentioning here that the actual rotational velocity of $\mathrm{L}$ dwarfs cannot be determined from their projected rotational velocity unless the inclination angle is known. Figure 6 shows that the model with mean particle size $3.0 \mu \mathrm{m}$ and polytropic index $n=1.5$ (nonrelativistic completely degenerate polytropic distribution) can explain the observed polarization of 2MASS $\mathrm{J} 0141+18$ (L4.5) and 2MASS J0144-07 (L5.0) if their rotational velocities are the same as that of Kelu $1\left(60 \mathrm{~km} \mathrm{~s}^{-1}\right)$.

As mentioned earlier, the object 2MASS J0036+1821 (L3.5) is observed in three different wavelength regions, and the degree of polarization is found to decrease substantially with the increase in wavelength. This trend strongly supports the presence of dust in the atmosphere of L dwarfs, as it is very much unlikely that any other mechanisms such as the presence of magnetic field can explain this. Figure 7 presents the degree of polarization as a function of wavelength for an L3.5 object with the polytropic index $n=1.5$ and the rotational velocity $v=15 \mathrm{~km} \mathrm{~s}^{-1}$. It is obvious from the observed data at three different wavelengths that the atmosphere of 2MASS J0036+1821 should have grains of submicron size. We find the best fit of the three observed data with the mean particle diameter $d_{0}=0.43 \mu \mathrm{m}$. Larger grain size would have made the polarization peak at longer wavelength.

The degree of polarization increases with the decrease in the polytropic index because with $n=1.0$, the oblateness of the object is higher than that with $n=1.5$ for the same rotational velocity and surface gravity. On the other hand, Sengupta (2003) showed that the oblateness decreases with the increase in surface gravity yielding less polarization.

As mentioned above, we have considered only forsterite in our models as it is very common in the atmosphere of L-type dwarfs with solar metallicity. This is because of large abundances of $\mathrm{Mg}, \mathrm{Si}$, and $\mathrm{O}$. However, gehlenite, enstatite, etc., should also be present in the atmosphere in fairly high abundance. The condensation curve presented in $\mathrm{C} 03$ shows that the base of gehlenite is situated much deeper in the atmosphere than that of forsterite yielding into a larger vertical size of the cloud. Further, iron with higher refractivity may undercircle most of the silicate clouds. Inclusion of all these will lead to substantial increase in degree of polarization, and therefore, much larger grain size may be needed in order to explain the observed degree of polarization.

Last, multiple scattering will lead to much less polarization, and therefore in order to fit the observed polarization one has to either consider much higher oblateness of the objects or increase the grain number density substantially. An increase in grain number density needs smaller grain size, which may contradict the present theoretical understanding on the nature and formation of dust grains. On the other hand, differential photometric

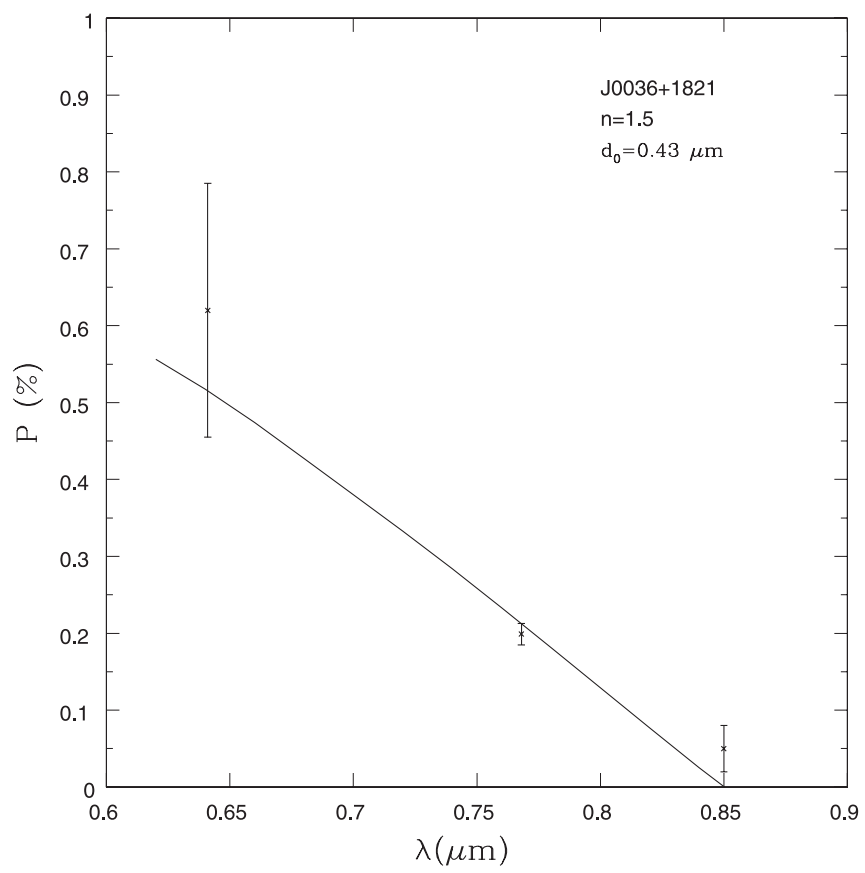

FIG. 7.-Degree of polarization as a function of wavelength with the mean grain diameter $d_{0}=0.43 \mu \mathrm{m}$ and polytropic index $n=1.5$. The observed polarization of the L dwarf 2MASS J0036+1821 at three different wavelengths is fitted with this model. The rotational velocity of this object is taken as $V=15 \mathrm{~km} \mathrm{~s}^{-1}$.

observations of several L dwarfs could not detect any nonperiodic variability of many objects that show high polarization. This indicates optically thin dust shells, and therefore polarization by single dust scattering is quite reasonable.

\section{CONCLUSIONS}

We have investigated the optical linear polarization from $\mathrm{L}$ dwarfs of different spectral type by considering single dust scattering. Forsterite is considered to be the dominant species among the various condensates that could be present in the atmosphere of $\mathrm{L}$ dwarfs. The location of the cloud base and the cloud deck is determined from the condensation curve for forsterite and the atmospheric temperature-pressure profiles of different spectral types. The surface gravity is fixed at $10^{5} \mathrm{~cm} \mathrm{~s}^{-2}$, and a wide range of rotational velocity is considered. It is found that the degree of linear polarization decreases from hotter to cooler L dwarfs. However, L dwarfs with effective temperature greater than $2200 \mathrm{~K}$ should not show a detectable amount of polarization due to dust scattering, as either most of the dust would evaporate from the atmosphere or condensation would not be favored at such high temperature. It is found that the mean diameter of grains that is consistent with the observed polarization should not exceed a few microns, although a small amount of very large grains at the base of the cloud for comparatively cooler L dwarfs may well be accommodated. However, the observational data of 2MASS J0036+1821 clearly indicates the presence of submicron-sized grains. Further polarimetric observation at the optical and at other wavelengths would provide convincing information on the properties and distribution of dust in the atmosphere of $L$ dwarfs.

We are thankful to the referee for several valuable comments and suggestions. 


\section{REFERENCES}

Ackerman, A. S., \& Marley, M. S. 2001, ApJ, 556, 872

Allard, F., Hauschildt, P. H., Alexander, D. R., Tamanai, A., \& Schweitzer, A. 2001, ApJ, 556, 357

Barnes, J. W., \& Fortney, J. J. 2003, ApJ, 588, 545

Basri, G. 1999, Proc. American Astron. Soc., 194, 8208

Basri, G., et al. 2000, ApJ, 538, 363

Burrows, A., Hubbard, W. B., Lunine, J. I., \& Liebert, J. 2001, Rev. Mod. Phys., 73, 719

Burrows, A., \& Sharp, C. M. 1999, ApJ, 512, 843

Chabrier, G., Baraffe, I., Allard, F., \& Hauschildt, P. 2000, ApJ, 542, 464

Chandrasekhar, S. 1933, MNRAS, 93, 539

Cooper, C. S., Sudarsky, D., Milsom, J. A., Lunine, J. I., \& Burrows, A. 2003, ApJ, 586, 1320 (C03)

Dolginov, A. Z., Gnedin, Yu. N., \& Silant'ev, N. A. 1995, Propagation and Polarization of Radiation in Cosmic Media (Basel: Gordon \& Breach)

Golimowski, D. A., et al. 2004, AJ, 127, 3516

Hubbard, W. B. 1984, Planetary Interiors (New York: Van Nostrand Reinhold)

Kirkpatrick, J. D., et al. 1999, ApJ, 519, 802

Knapp, G. R., et al. 2004, AJ, 127, 3553

Marley, M., Saumon, D., Guillot, T., Freedman, R., Hubbard, W. B., Burrows, A., \& Lunine, J. I. 1996, Science, 272, 1919
Menard, F., Delfosse, X., \& Monin, J. 2002, A\&A, 396, L35

Murray, C. D., \& Dermott, S. F. 2000, Solar System Dynamics (New York: Cambridge Univ. Press)

Saumon, D., et al. 2000, ApJ, 541, 374

Scott, A., \& Duley, W. W. 1996, ApJS, 105, 401

Sengupta, S. 2003, ApJ, 585, L155

Sengupta, S., \& Krishan, V. 2001, ApJ, 561, L123

Simmons, J. F. L. 1982, MNRAS, 200, 91

Stephens, D. C., Marley, M. S., Noll, K. S., \& Chanover, N. 2001, ApJ, 556, L97

Tsuji, T. 2005, ApJ, 621, 1033

Tsuji, T., Nakajima, T., \& Yanagisawa, K. 2004, ApJ, 607, 511

Tsuji, T., Ohnaka, K., Aoki, W., \& Nakajima, T. 1996, A\&A, 308, L29

van de Hulst, H. C. 1957, Light Scattering by Small Particles (New York: Wiley)

Vrba, F. J., et al. 2004, AJ, 127, 2948

Woitke, P., \& Helling, Ch. 2003, A\&A, 399, 297 2004, A\&A, 414, 335

Zapatero Osorio, M. R., Caballero, J. A., \& Bejar, V. J. S. 2005, ApJ, 621, 445 